\title{
Generating Traffic Time Series Based on Generalized Cauchy Process
}

\author{
Ming $\mathrm{Li}^{1}$, S.C. $\mathrm{Lim}^{2}$, and Huamin Feng ${ }^{3}$ \\ ${ }^{1}$ School of Information Science \& Technology, East China Normal University, \\ Shanghai 200062, PR. China \\ mli@ee.ecnu.edu.cn, ming_lihk@yahoo.com \\ ${ }^{2}$ Faculty of Engineering, Multimedia University, 63100 Cyberjaya, Selanger, Malaysia \\ sclim@mmu.edu.my \\ ${ }^{3}$ Key Laboratory of Security and Secrecy of Information, Beijing Electronic Science and Tech \\ ology Institute, Beijing 100070, PR. China \\ fenghm@besti.edu.cn
}

\begin{abstract}
Generating traffic time series (traffic for short) is important in networking, e.g., simulating the Internet. In this aspect, it is desired to generate a time series according to a given correlation structure that may well reflect the statistics of real traffic. Recent research of traffic modeling exhibits that traffic is well modeled by a type of Gaussian process called the generalized Cauchy (GC) process indexed by two parameters that separately characterize the self-similarity (SS), which is local property described by fractal dimension $D$, and long-range dependence (LRD), which is a global feature that can be measured by the Hurst parameter $H$, instead of using the linear relationship $D=2-H$ as that used in traditional traffic model with a single parameter such as fractional Gaussian noise (FGN). This paper presents a computational method to generate series based on the correlation form of GC process indexed by 2 parameters. Hence, the present model can be used to simulate realizations that flexibly capture the fractal phenomena of real traffic for both short-term lags and long-term lags.
\end{abstract}

Keywords: Random data generation, network traffic, self-similarity, long-range dependence, generalized Cauchy process.

\section{Introduction}

Network traffic is a type of fractal series with both (local) self-similarity (SS) and long-range dependence (LRD). Hence, it is a common case to exhibit fractal phenomena of time series, see e.g. [1], [2] and references therein. Its simulation is greatly desired in the Internet communications [3]. Conventional method to generate random series is either based on a given probability density function, see e.g. [4], or a given power spectrum, see e.g. [5]. For traffic simulation, however, it is expected to accurately synthesize data series according to a predetermined correlation structure [3]. This is because not only the autocorrelation function (ACF) of traffic with LRD is an ordinary function while the power spectrum of a series with LRD is a generalized function but also ACF of arrival traffic greatly impacts the performances of queuing 
systems [6]. In addition, performance analysis desires to accurately know how one packet arriving at time $t$ statistically correlates to the other arriving at $t+\tau$ apart in future as remarked in [3, First sentence, Subparagraph 4, Paragraph 2, Section 6.1]. Therefore, this paper focuses on correlation based computational method.

As known, the statistics of synthesized traffic relies on traffic correlation model used in simulation. FGN with a single parameter is a widely used traditional traffic model, see e.g. [7], [8], [9]. Hence, traditional methods to synthesize traffic are based on FGN with a single parameter, see e.g. [10], [11], [12], [13], [14]. The realizations based on those methods, therefore, only have the statistical properties of FGN with a single parameter, which characterizes SS and LRD by the linear relationship $D=2-H$. Recall that SS and LRD are two different concepts, see e.g. [15], [16], [17], [18]. In fact, let $X(t)$ be traffic series. Then, $X(t)$ being of SS with SS index $\kappa$ means

$$
X(a t) \triangleq a^{\kappa} X(t), a>0,
$$

where $\triangleq$ denotes equality in finite joint finite distribution. On the other hand, $X(t)$ is of LRD if its ACF, $r(\tau)$, is non-summable. That is, $r(\tau)$ follows power law given by

$$
r(\tau) \sim c \tau^{-\beta}(\tau \rightarrow \infty), c>0,0<\beta<1 .
$$

In principle, $D$ and $H$ can be measured independently [15-18]. The former can be used to characterize SS while the later LRD. In the case of FGN introduced by [19], however, they are linearly related. ACF of FGN in the discrete case is given by

$$
\frac{\sigma^{2}}{2}\left(|| \tau|+1|^{2 H}-2|\tau|^{2 H}+|| \tau|-1|^{2 H}\right)
$$

where $\sigma^{2}=\frac{\Gamma(2-H) \cos (\pi H)}{\pi H(2 H-1)}[1]$, [31]. The case $0.5<H<1$ corresponds to LRD, $0<H<0.5$ implies short-range dependence (SRD). FGN reduces to standard white noise when $H=0.5$. FGN with a single parameter characterizes both SS and LRD of traffic by $H$, or equivalently $D$.

The limitation of single-parameter FGN in traffic modeling has been noticed as can be seen from the discussions in [9, Last sentence, Paragraph 4, §7.4], which stated that "it might be difficult to characterize the correlations over the entire traffic traces with a single Hurst parameter". As a matter of fact, [20] mentioned the shortcoming that the single parameter model, e.g., FGN, does not well fit series for short-term lags.

Recently, [16] introduced a class of stationary Gaussian processes indexed by two parameters, which separately characterizes SS and LRD of fractal time series. That model is in the Cauchy class since it can be regarded as extension of the generalized Cauchy (GC) process used in geostatistics [21]. In this regard, our recent papers [17], [18], [22] provided theoretical analysis with demonstrations and comparisons using real-traffic series to show that 2-parameter model is far agreement with real traffic in comparison with single parameter model such as FGN. In addition, our early work [14] gave a correlation-based method for the generation of LRD series with single parameter. Nevertheless, reports regarding the simulation of time series obeying the 
GC model are rarely seen, to our best knowledge, letting along the simulation of traffic series. In this paper, we substantially extend our previous work [14] based on GC process model [17] to synthesize traffic series with 2 parameters so that the synthesized series has the property that SS and LRD are decoupled.

The remaining article is organized as follows. Section 2 briefs the concept of GC processes. Section 3 gives the computational model of traffic generation based on the Cauchy correlation model. Section 4 illustrates the demonstrations. Finally, Section 5 concludes the paper.

\section{Concept of GC Process}

$X(t)$ is called a GC process if it is a stationary Gaussian centred process with the following ACF:

$$
r(\tau)=E[X(t+\tau) X(t)]=\left(1+\tau^{\alpha}\right)^{-\beta / \alpha}, \tau>0,
$$

where $0<\alpha \leq 2$, and $\beta>0,[16],[17],[18]$.

Note that $r(\tau)$ is positive-definite for the above ranges of $\alpha$ and $\beta$, and it is a completely monotone for $0<\alpha \leq 1, \beta>0$. When $\alpha=2$ and $\beta=1$, one gets the usual Cauchy process.

Clearly the GC process satisfies the LRD property for $\beta<1$ since

$$
\int_{0}^{\infty} r(\tau) d \tau=\int_{0}^{\infty}\left(1+|\tau|^{\alpha}\right)^{-\beta / \alpha} d \tau=\infty \text { if } \beta<1 .
$$

We note that the GC process is locally self-similar as can be seen from the following. As a matter of fact, it is a Gaussian stationary process and it is locally self-similar of order $\alpha$ since its ACF satisfies for $\tau \rightarrow 0$,

$$
r(\tau)=1-\frac{\beta}{\alpha}|\tau|^{\alpha}\left\{1+O\left(|\tau|^{\gamma}\right)\right\}, \quad \gamma>0
$$

The above expression is equivalent to the following more commonly used definition of locally self-similarity of a Gaussian process.

$$
X(t)-X(a \tau) \triangleq a^{\kappa}[X(t)-X(\tau)], \quad \tau \rightarrow 0 .
$$

The equivalence can be shown by noting that for $\tau_{1}$ and $\tau_{2} \rightarrow 0$, (6) gives for

$$
\begin{aligned}
& \kappa=\alpha / 2 \\
& E\left[\left(X\left(t+b \tau_{1}\right)-X(t)\right)\left(X\left(t+b \tau_{2}\right)-X(t)\right)\right]=\frac{\beta}{\alpha}\left[\left|b \tau_{1}\right|^{\alpha}+\left|b \tau_{2}\right|^{\alpha}-\left|b\left(\tau_{1}-\tau_{2}\right)\right|^{\alpha}\right] \\
& =\frac{\beta b^{\alpha}}{\alpha}\left(\left|\tau_{1}\right|^{\alpha}+\left|\tau_{2}\right|^{\alpha}-\left|\tau_{1}-\tau_{2}\right|^{\alpha}\right)=E\left\{\left[b^{\alpha / 2}\left(X\left(t+\tau_{1}\right)-X(t)\right)\right]\left[b^{\alpha / 2}\left(X\left(t+\tau_{2}\right)-X(t)\right)\right]\right\} .
\end{aligned}
$$

In order to determine the fractal dimension of the graph of $X(t)$, we consider the local property of traffic. The fractal dimension $D$ of a locally self-similar traffic of order $\alpha$ is given by (see [17], [18], [23]). 


$$
D=2-\frac{\alpha}{2}
$$

From (2), therefore, one has

$$
H=1-\frac{\beta}{2}
$$

From (8) and (9), we see that $D$ and $H$ may vary independently. $X(t)$ is of LRD for $\beta \in(0,1)$ and SRD for $\beta>1$. Thus, we have the fractal index $\alpha$ which determines the fractal dimension, and the index $\beta$ that characterizes the LRD. In the end of this section, we discuss estimates of $D$ and $H$ of a GC process for the purpose of completing the description of GC processes though the focus of this paper does not relate to their estimators.

There are some techniques that are popularly used to estimate $D$, such as boxcounting, spectral, and variogram-based methods, see e.g. [15], [24], [25]. Nevertheless, some of the more popular methods, e.g., box-counting, suffer from biases [26]. The method worth noting is called the variogram estimator that was explained in [27]. Denote $\gamma(d)$ the observed mean of the square of the difference between two values of the series at points that are distance $d$ apart. Then, this estimator has the scaling law given by

$$
\log \gamma(d)=\text { constant }+\alpha \log d+\text { error for } d \rightarrow 0 \text {. }
$$

The above scaling law is suitable for stationary processes satisfying $1-r(\tau) \sim|\tau|^{-\alpha}$ for $\tau \rightarrow 0$. The variogram estimator of $D$ is expressed by $\hat{D}=2-\hat{\alpha} / 2$, where $\hat{\alpha}$ is the slope in a log-log plot of $\gamma(d)$ versus $d$.

The reported estimators of $H$ are rich, such as $R / S$ analysis, maximum likelihood method, and so forth [2], [15], [28]. The method worth mentioning is called the detrended fluctuation analysis introduced in [29], [30]. With this technique, a series is partitioned into blocks of size $m$. Within each block, least-square fitting is used. Denote $v(m)$ the average of the sample variances. Then, the detrended fluctuation analysis is based on the following scaling law.

$$
\log v(m)=\text { constant }+(2-\beta) \log m+\text { error for } m \rightarrow \infty .
$$

The above is applicable for stationary processes satisfying (2), see [28] for details. The estimate $\hat{H}$ of $H$ is half the slope in a log-log plot of $v(m)$ versus $m$.

\section{Computational Model}

Let $w(t), W(\omega)$ and $S_{w}(\omega)$ be a white noise function, its spectrum and the power spectrum, respectively. Then, $W(\omega)=F[w(t)]$ and $S_{w}(\omega)=W \bar{W}=$ Constant, where $\bar{W}$ is 
the complex conjugation of $W$ and $F$ is the operator of Fourier transform. Suppose $w(t)$ is the unit white noise. Then, $S_{w}(\omega)=1$.

Let $h(t)$ and $H(\omega)$ respectively be the impulse function and system function of a linear filter, which we call simulator in this paper. Denote $y(t)$ the response under the excitation of $w(t)$. Then, $y=w * h$, where $*$ means the operation of convolution. Denote $S_{y}(\omega)$ the power spectrum of $y$. Then, under the excitation of $w$, one has $S_{y}(\omega)=|H(\omega)|^{2}$. Let $y$ be the GC process $X$. Then, $|H(\omega)|=\sqrt{S_{X}(\omega)}$.

Denote $\psi(\omega)$ the phase function of $H(\omega)$. Then, $H(\omega)=\sqrt{S_{X}(\omega)} e^{-j \psi(\omega)}$, where

$$
S_{X}(\omega)=F\left[\left(1+|t|^{\alpha}\right)^{-\beta / \alpha}\right] \text {. }
$$

Without losing the generality, let $\psi(\omega)=2 n \pi(n=0,1, \cdots)$. Therefore, the impulse function of the simulator to generate traffic following GC processes under the excitation of white noise is

$$
h(t)=F^{-1}\left\{\left[F\left(1+|t|^{\alpha}\right)^{-\beta / \alpha}\right]^{0.5}\right\},
$$

where $F^{-1}$ is the inverse of $F$. Consequently, the output of simulator, i.e., the synthesized traffic obeying GC processes is given by

$$
X(t)=w(t) * F^{-1}\left\{\left[F\left(1+|t|^{\alpha}\right)^{-\beta / \alpha}\right]^{0.5}\right\} .
$$

Expressing $\alpha$ and $\beta$ by $D$ and $H$, we have

$$
X(t)=w(t) * F^{-1}\left\{\left[F\left(1+|t|^{4-2 D}\right)^{-\frac{1-H}{2-D}}\right]^{0.5}\right\} .
$$

In the above, $w(t)=F^{-1}[W(\omega)]$, where $W(\omega)=e^{j \vartheta(\omega)}$, where $\theta$ is a real random function with arbitrary distribution. In practice, traffic is band-limited. Thus, let

$$
W(\omega)=\left\{\begin{array}{c}
e^{j \phi(\omega)},|\omega| \leq \omega_{c}, \\
0, \text { otherwise }
\end{array}\right.
$$

where $\phi(\omega)$ is a real random function with arbitrary distribution and cutoff frequency $\omega_{c}$ is such that it completely covers the band of the traffic of interest.

In the discrete case, $w(n)=\operatorname{IFFT}[W(\omega)]$, where IFFT represents the inverse of FFT (fast Fourier transform). Fig. 1 indicates the computation procedure. 


\section{Case Study}

Simulated realizations are shown in Figs. 2-3. Due to the advantage of the separated characterizations of $D$ and $H$ by using the Cauchy correlation model, we can observe the distinct effects of $D$ and $H$. In Fig. 2, the Hurst parameter $H$ is constant 0.75 but the fractal dimension $D$ decreases, $(D=1.95$ and 1.20). Comparing Fig. 2 (a) and (b), we can see the realization in Fig. 2 (a) is rough while that in Fig. 2 (b) smooth.

In Fig. 3, fractal dimension $D$ is constant 1 while $H$ decreases $(H=0.95$ and 0.55). From Fig. 3, we can evidently see the stronger persistence (i.e., stronger LRD) in (a) than that in (b).

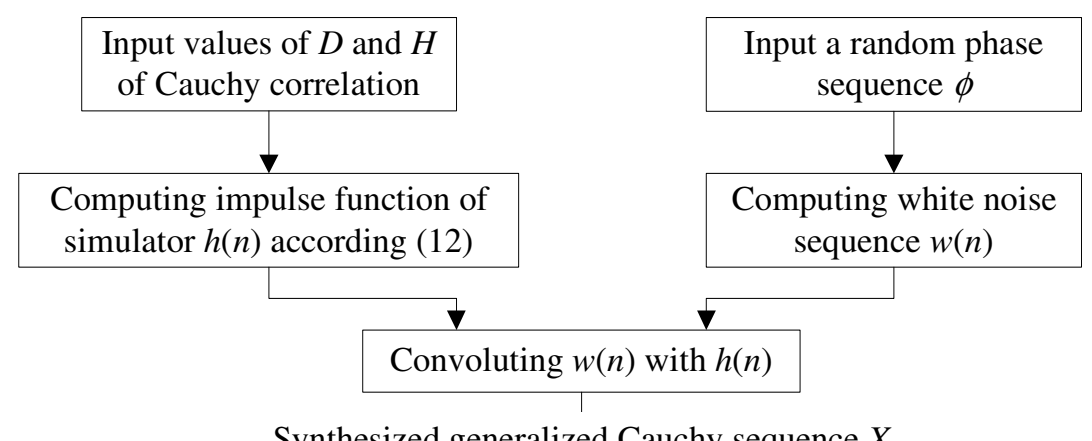

Synthesized generalized Cauchy sequence $X$

Fig. 1. Computation flow chart

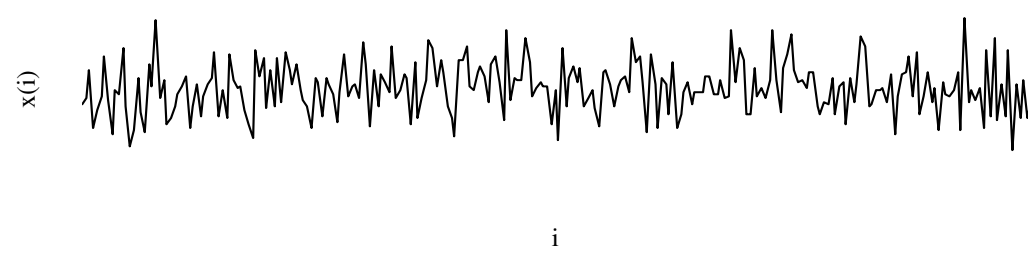

(a)
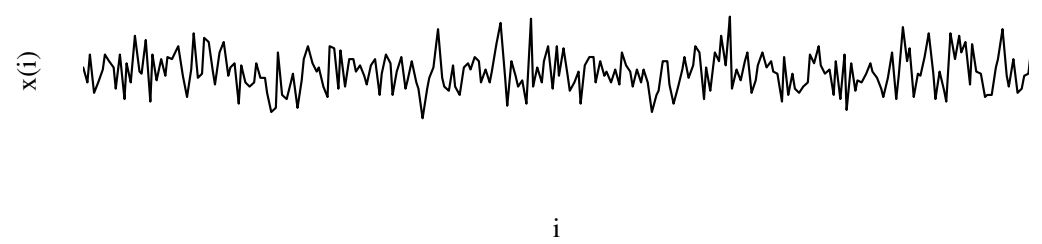

(b)

Fig. 2. Realizations. (a) $D=1.95, H=0.75$. (b) $D=1.20, H=0.75$. 


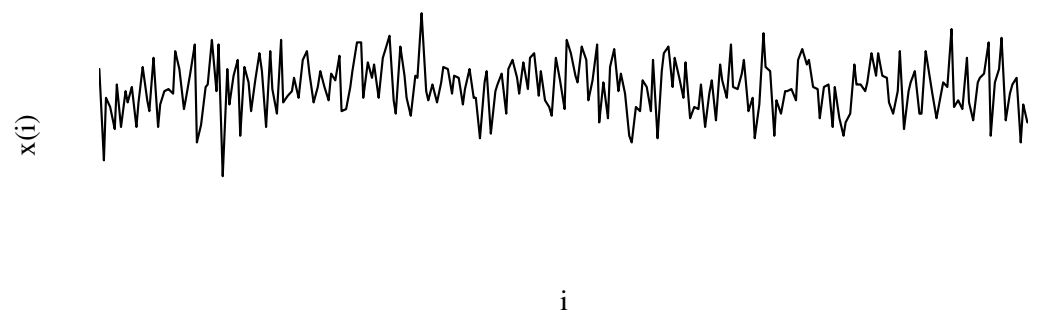

(a)

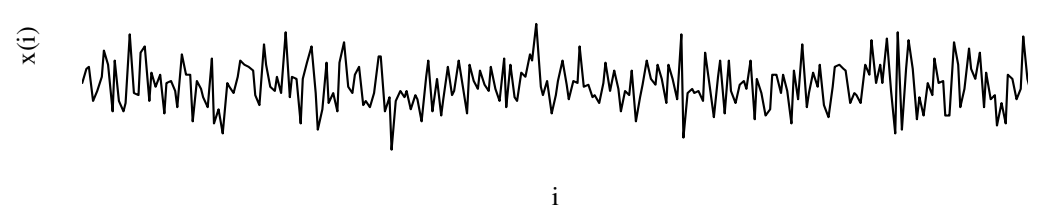

(b)

Fig. 3. Realizations. (a). $H=0.95, D=1$. (b). $H=0.55, D=1$.

\section{Conclusions}

We have given a computational model to generate traffic with separate parametrization of the self-similarity property and long-range dependence based on the correlation model of the generalized Cauchy processes. Since this correlation model can separately characterize the fractal dimension and the Hurst parameter of a process, the present method can be used to simulate realizations that have the same long-range dependence with different fractal dimensions (i.e., different burstinesses from the view point of networking). On the other hand, we can synthesize realizations that have the same fractal dimension but with different long-range dependencies. Hence it provides a flexible way to simulate realizations of traffic. These are key advantages of the computational model presented.

\section{Acknowledgements}

This work was supported in part by the National Natural Science Foundation of China under the project grant numbers 60573125 and 60672114 , by the Key Laboratory of Security and Secrecy of Information, Beijing Electronic Science and Technology Institute under the project number KYKF 200606 of the open founding. SC Lim would like to thank the Malaysia Ministry of Science, Technology and Innovation for the IRPA Grant 09-99-01-0095 EA093, and Academy of Sciences of Malaysia for the Scientific Advancement Fund Allocation (SAGA) P 96c. 


\section{References}

1. Mandelbrot, B. B.: Gaussian Self-Affinity and Fractals. Springer (2001)

2. Beran, J.: Statistics for Long-Memory Processes. Chapman \& Hall (1994)

3. Paxson, V., Floyd, S.: Why We Don't Know How to Simulate the Internet. Proc., Winter Simulation Conf. (1997) 1037-1044

4. Press, W. H., Teukolsky, S. A., Vetterling, W. T., Flannery, B. P.: Numerical Recipes in C: the Art of Scientific Computing. $2^{\text {nd }}$ Edition, Cambridge University Press (1992)

5. Li, M.: Applied Mathematical Modelling 29 (2005) 55-63

6. Livny, M., Melamed, B., Tsiolis, A. K.: The Impact of Autocorrelation on Queuing Systems. Management Science 39 (1993) 322-339

7. Tsybakov, B., Georganas, N. D.: IEEE T. Information Theory 44 (1998) 1713-1725

8. Li, M., Zhao, W., Jia, W., Long, D. Y., Chi, C.-H.: Modeling Autocorrelation Functions of Self-Similar Teletraffic in Communication Networks based on Optimal Approximation in Hilbert Space. Applied Mathematical Modelling 27 (2003) 155-168

9. Paxson, V., Floyd, S.: IEEE/ACM T. Networking 3 (1995) 226-244

10. Paxson, V.: Fast Approximate Synthesis of Fractional Gaussian Noise for Generating SelfSimilar Network Traffic. Computer Communication Review 27 (1997) 5-18

11. Jeong, H.-D. J., Lee, J.-S. R., McNickle, D., Pawlikowski, P.: Simulation. Modelling Practice and Theory 13 (2005) 233-256

12. Ledesma, S., Liu, D.: Computer Communication Review 30 (2000) 4-17

13. Garrett, M. W., Willinger, W.: Analysis, Modeling and Generation of Self-Similar VBR Traffic. Proc., ACM SigComm'94, London (1994) 269-280

14. Li, M., Chi, C.-H.: A Correlation-Based Computational Method for Simulating LongRange Dependent Data. Journal of the Franklin Institute 340 (2003) 503-514

15. Mandelbrot, B. B.: The Fractal Geometry of Nature. W. H. Freeman (1982)

16. Gneiting, T., Schlather, M.: Stochastic Models That Separate Fractal Dimension and Hurst Effect. SIAM Review 46 (2004) 269-282

17. Li, M., Lim, S. C.: Modeling Network Traffic Using Cauchy Correlation Model with Long-Range Dependence. Modern Physics Letters B 19 (2005) 829-840

18. Lim, S. C., Li, M.: Generalized Cauchy Process and Its Application to Relaxation Phenomena. Journal of Physics A: Mathematical and General 39 (12) (2006) 2935-2951

19. Mandelbrot, B. B., van Ness, J. W.: Fractional Brownian Motions, Fractional Noises and Applications. SIAM Review 10 (1968) 422-437

20. Kaplan, L. M., Kuo C.-C. J.: IEEE T. Signal Processing 42 (1994) 3526-3530

21. Chiles, J-P., Delfiner, P.: Geostatistics, Modeling Spatial Uncertainty (Wiley) (1999)

22. Li, M.: Modeling Autocorrelation Functions of Long-Range Dependent Teletraffic Series based on Optimal Approximation in Hilbert Space-a Further Study. Applied Mathematical Modelling 31 (3) (2007) 625-631

23. Kent, J. T., Wood, A. T. A.: J. R. Statit. Soc. B 59 (1997) 579-599

24. Dubuc, B., Quiniou, J. F., Roques-Carmes, C., Tricot, C., Zucker, S. W.: Phys. Rev. A 39 (1989) 1500-1512

25. Hall P., Wood, A.: Biometrika 80 (1993) 246-252

26. Taylor, C. C., Taylor, S. J.: J. Roy. Statist. Soc. Ser. B 53 (1991) 353-364

27. Constantine, A. G., Hall, P.: J. Roy. Statist. Soc. Ser. B 56 (1994) 97-113

28. Taqqu, M. S., Teverovsky, V., Willinger, W.: Fractals 3 (1995) 785-798

29. Peng, C.-K., Buldyrev, S. V., Havlin, S., Simons, M., Stanley, H. E., Goldberger, A. L.: Mosaic Organization of DNA Nucleotides. Phys. Rev. E 49 (1994) 1685-1689

30. Kantelhardt, J. W., et al.: Phys. A 295 (2001) 441-454

31. Li, M., Lim, S. C.: A Rigorous Derivation of Power Spectrum of Fractional Gaussian Noise. Fluctuation and Noise Letters 6 (4) 2006, C33-36 